\title{
Flame Retardants Finish on Hand Mittens of Woollen Fabs
}

\author{
Mrs. D. Anitarachel ${ }^{1}$, Abilash Kumar. B ${ }^{2}$ \\ ${ }^{1}$ Assistant Professors, ${ }^{2}$ B.Sc Sudent \\ ${ }^{1}$ Department of Apparel Manufacturing and Merchandising, \\ ${ }^{1}$ Research Scholar Department of Rural Industries and Management, \\ ${ }^{1}$ Gandhigram Rural Institute-Deemed University, Gandhigram, Dindigul, Tamil Nadu, India \\ ${ }^{2}$ NIFT-TEA College Of Knitwear Fashion, Tirupur, Tamil Nadu, India
}

\begin{abstract}
Processes on fire resistance in the kitchen linen yields of $100 \%$ Woollen Fabrics were discussed. Evaluating the Woollen fabrics on kitchen products such as dining category on fire resistance are it is analyzed based on the result of using some techniques and usage of softeners the Products are Woollen Glove, Pot Holder and Oven Mitten, Flame retardant chemicals \& natural minerals. There are many dangers in using cloths without fire resistance or fire proof(flame retardant finishes)in kitchen linens, and then selecting of fire resisted in cloth is worse and so by giving numerous tests with softeners such as oils, wax, tallow, soda ash, ammonia \& organic phosphorous with Flame resisting chemicals.
\end{abstract}

To obtain the kitchen linens sets such as hand towels, place mats, runners, napkins, pot holders, apron, oven mitts, table cloth, and chair pads with the softeners treatment.

Keywords: Kitchen Products, Flame retardant finish, Safety purpose, Gloves, Woollen Glove, Pot Holder and Oven Mitten

\section{INTRODUCTION}

The textiles on the one hand protect us from external agencies in our daily life and pose a significant fire hazards may appear. The flame resistance of textile is of importance for improvement of the safety characteristics of apparel, bedding, protective clothing, carpets, home furnishing, industrial fabrics which may be woven, knitted, tufted and non woven.

It is therefore a very important object of current research and development programs to make available flame retardant finishes which can be used on natural \& synthetic textiles and fabrics, especially on celluloses and cellulose synthetic blends, which involves the use of substantially non toxic, less volatile compounds and which leave the fabric with good handle, good physical strength properties. The flammability of cotton products is one of the major problems posed to scientists and technologists at the present time. Resistance to burning is a useful property that can be imparted to cotton fibers and fabrics. There is the potential for 2-3 million bales of cotton to be affected by flammability regulations and issues. The apparel portion of this is more than 1 million bales, with the remainder including categories such as home furnishing, bedding, tent cloth, carpets, aircraft and automobile interior fabrics and industrial fabrics.

Government regulations, insurance company requirements, building codes, and voluntary standards dictate where and when flame-resistant textiles must be used. Also in today's litigious environment, textile producers are becoming increasingly concerned with the liability to which they may be exposed if someone accuses their products of causing an injury or fatality.

Cotton and cellulosic textiles in general, while being among the most flammable of materials. Hazards from flammable fabrics were recognized for many centuries and repeated attempts have been made to cope with them. Cotton is the most commonly used of textile fibers. It is not surprising that most efforts in the field of flame retardancy were made on modifying the flaming properties of cotton fibers. There are currently several different types of chemical finishes that can be applied to cellulosic materials to impart flame retardant properties. Of these systems, only a few create finished fabrics that can belaundered and dry-cleaned without losing their flame retardant qualities. These treatments are generally referred to as "durable flame retardant finishes".

\subsection{AIMS \& OBJECTIVES:}

1.4 To produce a Woollen Glove, Pot Holder and Oven Mitten in all sizes.

2. To develop the flame resistance on the Woollen Glove, Pot Holder and Oven Mitten with Natural mineral.

3. To improve the safety characteristics on the Woollen Glove Products, Pot Holder and Oven Mitten.

\subsection{OVEN MITTS:}

Oven mitts can simply be pads of material or they can be single or double gloves. You may prefer one style over another. A double glove oven mitt can be useful if you often need to carry large hot dishes with both hands, while a small pad can be a better choice if you only want to be able to grab a small dish and move it quickly. You might want to buy a couple of different styles of oven mitts for different uses.

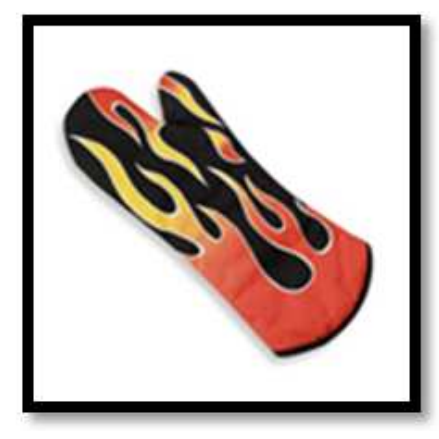

OVEN MITTS 
An oven mitt needs to be made from thick, durable, heatresistant material that will be able to protect your hands. It should also have a loop that you can use to hang it up in a convenient place.

\subsection{POT HOLDERS:}

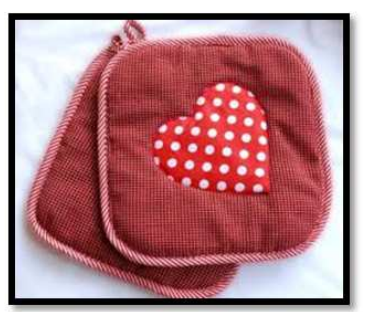

$>$ A pot-holder is a piece of textile or silicone used to hold hot kitchen cooking equipment, like pots and pans. They are made mostly of polyester and/or cotton. Another type of "pot holder" is a trivet.

A thick piece of quilted material or woven pad used to protect your hands from the heat of hot cookware, casseroles, or other hot items that cannot be handled with bare hands

\section{FLAME RETARDANT:}

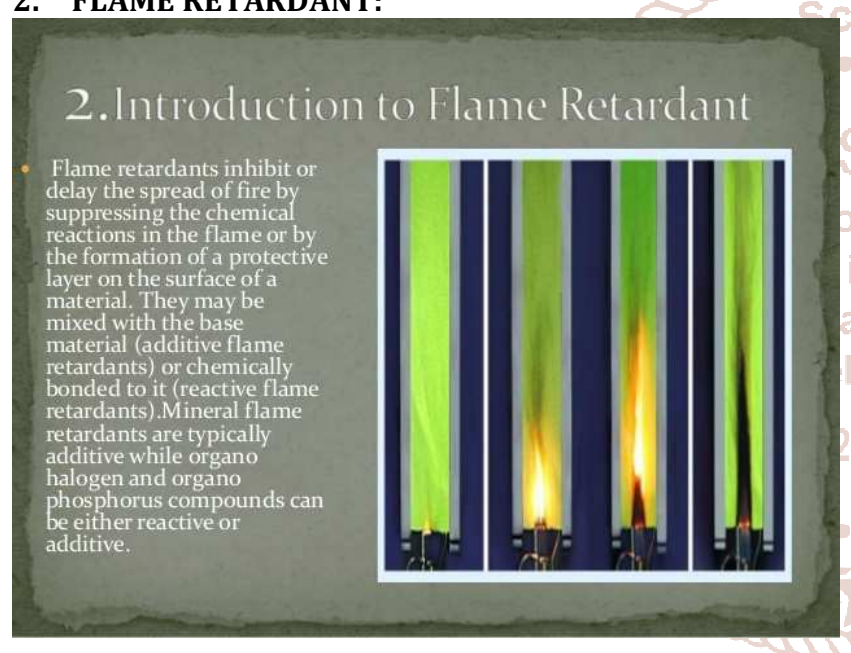

Flame or fire retardants are chemicals which can be added to combustible substrates, to give them a better protection to ignition. Depending on the type of flame retardant and substrate, this protection can be so good that it is even impossible to burn the substrate at all. Simply defined, flame retardants are materials that have the quality of inhibiting or resisting the spread of fire. Textile is highly ignitable and contributes to rapid fire spread. However, the ignitable property of a textile can be considerably reduced by any one of the three methods- by using inorganic materials such as Asbestos, Glass etc; by chemically treating the textile with Flame Retardant chemicals; and by modifying the polymer.
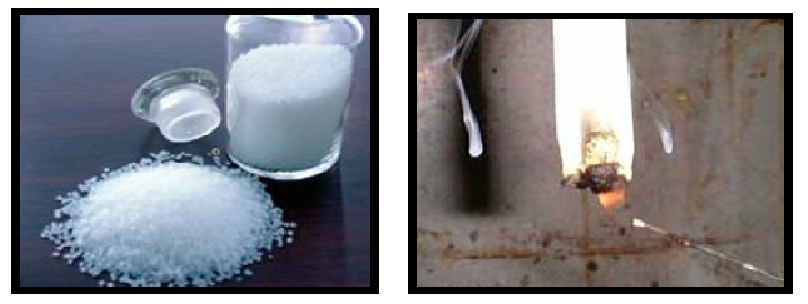

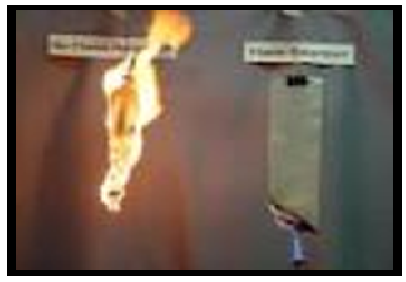

\subsection{FUNCTION OF A FLAME RETARDANT:}

Flame retardants are chemicals are applied to fabrics to inhibit or suppress the combustion process. They interfere with combustion at various stages of the process e.g. during heating, decomposition, ignition of flame spread. Fire is gas phase reaction. For a substance to burn, it must become a gas. As with any solid, a textile fabric exposed to a heat source experiences a temperature rise. If the temperature of the source (either radiative or gas flame) is high enough and the net rate of heat transfer to the fabric is great, pyrolytic decomposition of the fiber substrate will occur. The products of this decomposition include combustible gases, non combustible gases and carbonaceous char. The combustible gases mix with the ambient air and its oxygen. The mixture ignites, yielding a flame, when its composition and temperature are favorable. Part of the heat generated within the flame is transferred to the fabric to sustain the burning process and part is lost to the surroundings.

\section{PROCESSING:}

\subsection{PADDING BAKING PROCESS:}

The process flow of the method is: Padding, preliminary drying, baking after treatment. Its padding liquor is flame retardant solution, commonly be formed of flame retardant, cross-linking agent, catalyze, wetting agent and softener, either formulate become water solution or lattices proceed tidy.

\subsection{STEEP PARCH DRY FARAD:}

Process flow is: Steep?dryness? ?after treatment. It is hold fabric lay in flame retardant liquor suffer steep hour to it, take out and drying, at times flame retardant finish approve to dyeing with bath proceed.

\subsection{EXTRUSION COATING FARAD:}

It is number flame retardant interfusion resin within proceed process. warranty the different of mechanical equipment in to knife coating farad, molding extrusion coating farad and recycle extrusion coating farad.

\subsection{SPRAY METHOD:}

Put out of service conventional equipment art factitious thick curtain, large-scale rug and more merchandise, the flame retardant finish of the approve last of all in a single operation did hand spraying farad. Toward bulkiness surface have got figure, tufting, pile cottony fabric, used padding farad dispose surface fuzz figure came in for traumata to, commonly adopt series spray method.

\subsection{BENEFITS AND FEATURES:}

$>$ Imparts a soft handle.

$>$ Low hydroscopic property.

$>$ Shows no corrosion.

$>$ Causes little or no shade change.

$>$ Has good thermal stability, therefore drying is possible at higher temperatures.

$>$ Can be combined with most synthetic resins or binders, a preliminary check is advisable. 


\author{
Woollen fabric \\ $\downarrow$ \\ Manufacturing the Hand Glove \\ $\downarrow$ \\ Flame retardant Method \\ $\downarrow$ \\ Toxic (Halogen) Non-Toxic (Environmental Friendly Halogen Free) \\ $\downarrow$ \\ Steeping Extrusion\& coating Spraying Padding \& Baking TPHC (Tetrakis Hydroxy \\ methyl chloride) Proban Flame Retardant Treating with the Softening agents \\ $>$ Wax (Paraffin, Beeswax, Japan wax) \\ $>$ Oils (Glycerin, Palm oil) \\ $>$ Tallow (Vegetable, Mutton) \\ $>$ Soda ash \& Ammonia \\ $\downarrow$ \\ Treating with sulphate of ammonia\& ammonium chloride \& Organic Phosphorous \\ $\downarrow$ \\ Treating with boric acid \& Borax \& Flame retardant chemicals \\ $\downarrow$ \\ Adding catalyze\& wetting agent drying the kitchen products \\ $\downarrow$ \\ Kitchen linen products with flame resistance
}

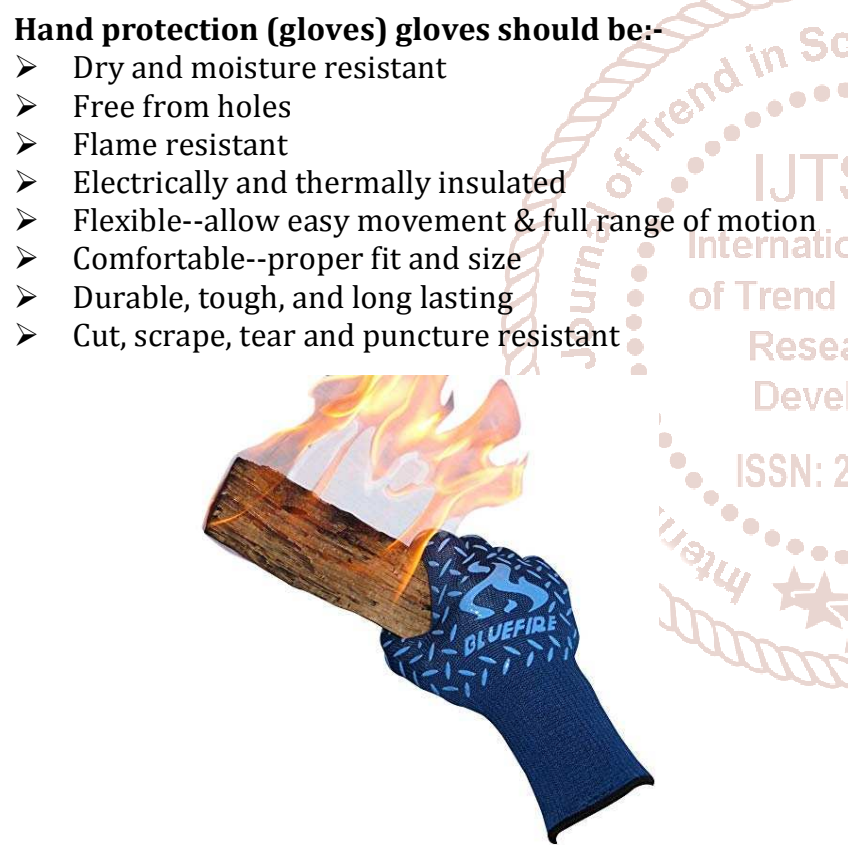

\subsection{BURN-IN JUSTIFICATION:}

First, we should be aware that burn-in testing is not necessary for all the products. In order to benefit from burnin testing, engineers should collect failure data to decide whether or not burn-in is necessary for the product. In this section, a general procedure for the quantification of a burnin test is given. It has the following three steps:

1. Times-to-failure data collection.

2. Initial data analysis.

3. Formal parametric data analysis.

\section{CONCLUSION:}

Hence to conclude that the flame retardant has been implemented towards the Woollen Glove, Pot Holder and Oven Mitten products, to ensure the safety purpose in kitchen. The flame retardant finish which was applied towards all the kitchen yield products with effectiveness of the study between the women's era. The attempt of flame retardant finish in the kitchen products was very good try and i ensure this kitchen products with flame retardant finish can be applied to all the home textile products to ensure the safety Purpose. I motivate the further project doer's to try this and implement in the market trends.

\section{REFERENCES}

[1] Wakelyn, P. J.1998. "Cotton and Flammability-Overview of New Development," American Dyestuff Reporter, 87(2): 13-21.

[2] Weil, E. D. 1995. in Kirk-Othmer Encyclopedia of Chemical Technology 4th Ed., Grayson M ed., 10: pp. 976-998.

[3] Lewin, M. and Sello, S.B. 1984. "Chemical processing of Fibers and Fabrics Functional Finishes" in Handbook of Fiber Science and Technology, Volume Two Part B Marcel Dekker, Inc. pp. 60-63, pp.78.

[4] Hendrix, J. E., Drake, G. L. and Barker, R. H. 1979. in Flame Retardancy of Polymeric Materials Volume Five, Kuryla, W. C. and Papa, A. H. eds., Marcel Dekker Inc. pp. 88.

[5] Isaacs, J. L. 1970. "The oxygen Index Flammability Test," J. Fire \& Flam. 1(1): 36- 42.

[6] Weil, E. D., Hirschler, M. M., Patel, N. G., Said, M. M., and Shakir, S. 1992. "Oxygen Index: Correlation to Other Fire Tests," Fire and Materials, 16(3):159-167.

[7] Wharton, R. K. 1981. "The Effect of Sample Size on the Burning Behavior of Thermoplastic Materials in the Critical Oxygen Index Test," Fire and Materials 5 (2): 7376.

[8] Wharton, R. K. 1984. "The performance of Various Testing Columns in Small- Scale Horizontal Burning Studies," Fire and Materials 8 (4): 177-182. 